\title{
Análise da aplicabilidade do Design of Experiments no Mercado Financeiro
}

\author{
Analysis of the applicability of Design of Experiments in the Financial Market
}

\author{
Rafael Munhoz Cardoso ${ }^{1 *}$, Érik Lonel Luciano${ }^{1}$, Prof. Dr. Alexandre Formigoni ${ }^{1}$, Profa. Dra. \\ Eliane Antonio Simões ${ }^{1}$, Prof. Dr. Rosinei Batista Ribeiro ${ }^{1}$
}

\begin{abstract}
RESUMO
O mercado financeiro, especificamente o mercado de ações, é um setor que absorve as consequências das instabilidades econômicas dos países, retendo riscos durante o seu processo de atuação. Nesse contexto, ferramentas de apoio vem se popularizando frente aos gestores como o Design of Experiments, que pode ser implantada no desenvolvimento e na otimização de sistemas, de processos e de produtos, porém, notase através de estudos que a mesma é subutilizada na área financeira. $O$ objetivo desta pesquisa é identificar a aplicabilidade do Design of Experiments em áreas distintas e os seus impactos. Para isso, serão analisadas as publicações dos últimos 10 anos, por região, idiomas e autores para obtenção dos registros mais relevantes no que se refere à utilização do DOE especificamente no mercado financeiro. Trata-se de um estudo qualitativo por recorrer às evidências de Revisão Sistemática da Literatura (RS). Espera-se, no entanto, por meio de análise bibliométrica, a incorporação de uma possível alternativa para o gerenciamento e análise de riscos no mercado financeiro.
\end{abstract}

Palavras-chave: Design of Experiments; Aplicabilidade; Gestão da Inovação; Análise de Probabilidades; Mercado Financeiro.

\begin{abstract}
The financial market, specifically the stock market, is a sector that absorbs the consequences of economic instabilities in countries, retaining risks during its operating process. In this context, support tools have become popular among managers such as Design of Experiments, which can be implemented in the development and optimization of systems, processes and products, however, it is noted through studies that it is underused in the financial area. The objective of this research is to identify the applicability of Design of Experiments in different areas and their impacts. For this, publications from the last 10 years will be analyzed, by region, languages and authors to obtain the most relevant records regarding the use of DOE specifically in the financial market. This is a qualitative study because it uses evidence from the Systematic Literature Review (SR). It is expected, however, through bibliometric analysis, the incorporation of a possible alternative for the management and analysis of risks in the financial market.
\end{abstract}

Keywords: Design of Experiments; Applicability; Innovation management; Probability Analysis; Financial market.

${ }^{1}$ Programa de Mestrado Profissional Gestão e Tecnologia em Sistemas Produtivos - Unidade de Pós-Graduação, Extensão e Pesquisa - Centro Estadual de Educação Tecnológica Paula Souza CEETEPS - São Paulo, Brasil

*E-mail: rafael.cardoso@cpspos.sp.gov.br 


\section{INTRODUÇÃO}

O mercado financeiro é setor permeado pelo fator incertezas, por lidar constantemente com as instabilidades econômicas e financeiras dos países e das empresas, na qual carece da necessidade de ferramentas para análise de probabilidades e cenários, possibilitando a introdução de variáreis que possam se materializar. Nesse sentido, uma das ferramentas que vem se popularizando frente aos gestores e se consolidando é o Design of Experiments (DoE).

Durakovic (2017) explica que Design of Experiments é uma ferramenta estatística utilizada no planejamento e condução de experimentos, bem como na análise e interpretação dos dados obtidos em ensaios de pesquisas distintas. Explica ainda que é uma ferramenta extremamente útil para as empresas, fazendo parte do seu processo de direcionamento em decisões.

Diante destas perspectivas e à medida que a procura pelo uso de ferramentas de experimentação aumenta tanto por empresas quanto pelos pesquisadores, seria realmente factível aplicar o Design of Experiments em área tão instável, como o mercado financeiro? E quais os impactos da aplicação no ramo de finanças? Esse questionamento norteará a análise da aplicabilidade da ferramenta na tentativa de evidenciar os benefícios dessa no processo de gerenciamento de riscos mediante as práticas macros sistêmicas que interferem diretamente nos resultados do mercado financeiro.

Este trabalho se justifica na medida que servirá de subsídio a empresa Social Flare.TECH na tomada de decisão para utilização do DOE em seus projetos futuros, onde ela tem como premissa, o investimento pesado em Pesquisa e Desenvolvimento de produtos com foco em Inteligência de mercado. Podemos ressaltar ainda, que os resultados obtidos poderão contribuir na análise de outras empresas e pesquisadores interessados na utilização do DoE nessa área.

Em suma, o objetivo geral deste trabalho é identificar a aplicabilidade do Design of Experiments em áreas afins e analisar e sintetizar os estudos recentes para visualização do impacto da ferramenta no que se refere à garantia de mais segurança e efetividade nas operações do ramo financeiro. Trata-se de uma pesquisa qualitativa, na qual se subsidiará através da Revisão Sistemática da Literatura. Espera-se que este estudo possa contribuir para identificar uma possível ferramenta de apoio aos profissionais na área de finanças. 


\section{FUNDAMENTAÇÃO TEÓRICA}

\section{Design of Experiments DoE - Projeto de Experimentos - Perspectiva Histórica}

Telford (2007), em seu trabalho, desenvolveu uma ordem cronológica sobre a perspectiva histórica do DOE, nas quais foram:

Inventada por Ronald A. Fisher nas décadas de 1920 e 1930 na Estação Experimental de Rothamsted, uma estação de pesquisa agrícola, localizada a 40 quilômetros ao norte de Londres. Em seu primeiro livro, Fisher mostrou como conclusões válidas que o DOE podem ser extraídas com eficiência de experimentos com flutuações naturais, como temperatura, condições do solo e precipitação, ou seja, na presença de variáveis desconfortáveis.

Embora o método de projeto experimental tenha sido usado pela primeira vez em um contexto agrícola, o método tem sido aplicado com sucesso nas forças armadas e na indústria desde a década de 1940.

W. Edwards Deming ensinou métodos estatísticos, incluindo design experimental para cientistas e engenheiros japoneses no início da década de 1950, numa época em que "Made in Japan" significava baixa qualidade. Genichi Taguchi, o mais conhecido desse grupo de cientistas japoneses, é famoso por seus métodos de melhoria de qualidade. Uma das empresas onde Taguchi aplicou seus métodos pela primeira vez foi a Toyota.

Desde o final dos anos 1970, a indústria dos Estados Unidos voltou a se interessar por iniciativas de melhoria da qualidade, agora conhecidas como programas de "Qualidade Total" e "Seis Sigma". O design experimental é considerado um método avançado nos programas Seis Sigma que foram pioneiros na Motorola e na GE.

\section{O método Design of Experiments (DOE)}

Whitford, (2018) explica que o Design of Experiments (DoE) é uma técnica para planejar experimentos e analisar as informações obtidas. A técnica permite utilizar um número mínimo de ensaios, nos quais vários parâmetros de pesquisa são variados de forma sistemática e simultânea para obter informações suficientes. Com base nos dados obtidos, um modelo matemático do processo estudado (por exemplo, a quantidade e o tempo de um componente de alimentação) é desenvolvido. 
Huairui Guo (2012) ressalta que a ferramenta não se limita à engenharia e que muitas histórias de sucesso podem ser encontradas em outras áreas.

Para Zahraee, S. M. et al. (2013), DoE pode ser visto como sendo composto por uma série de etapas: o planejamento, a execução do experimento e a análise dos dados experimentais coletados usando vários métodos estatísticos para tirar conclusões válidas e objetivas.

Segundo Arsalan Farooq, et al. (2016), cada DoE começa selecionando o sistema/processo e reconhecendo o problema de investigação. Explicam ainda que a declaração do problema leva ao estabelecimento dos objetivos com base nos quais o indicador de desempenho (variável de resposta) precisa ser definido.

A variável de resposta deve representar uma medida quantitativa do comportamento do sistema. Como etapa essencial em todo o processo, os fatores que afetam o indicador de desempenho e como eles são discretizados, o número de execuções experimentais e uma matriz adequada precisam ser definidos na segunda etapa. A terceira etapa abrange a execução do experimento de acordo com a matriz projetada e coleta de dados (FAROOQ, MUHAMMAD, ARSALAN ET AL, 2016).

\section{Principais usos do DOE}

Atualmente, os pesquisadores selecionam principalmente o DoE com base na importância assumida dos fatores e o número desejado de experimentos (A. Alafaghani, A. Qattawi, 2018; F. Kolivand, R. Rahmannejad, 2018, C. Shen, L. Wang, W. Cao, L. Qian, 2007). A Figura 1 apresenta uma adaptação do modelo geral de processo apresentado por Montgomery (2017) com os componentes usados nesse trabalho.

Figura 1 - Modelo geral de processo e suas entradas, fatores controláveis e não controleis e saídas.

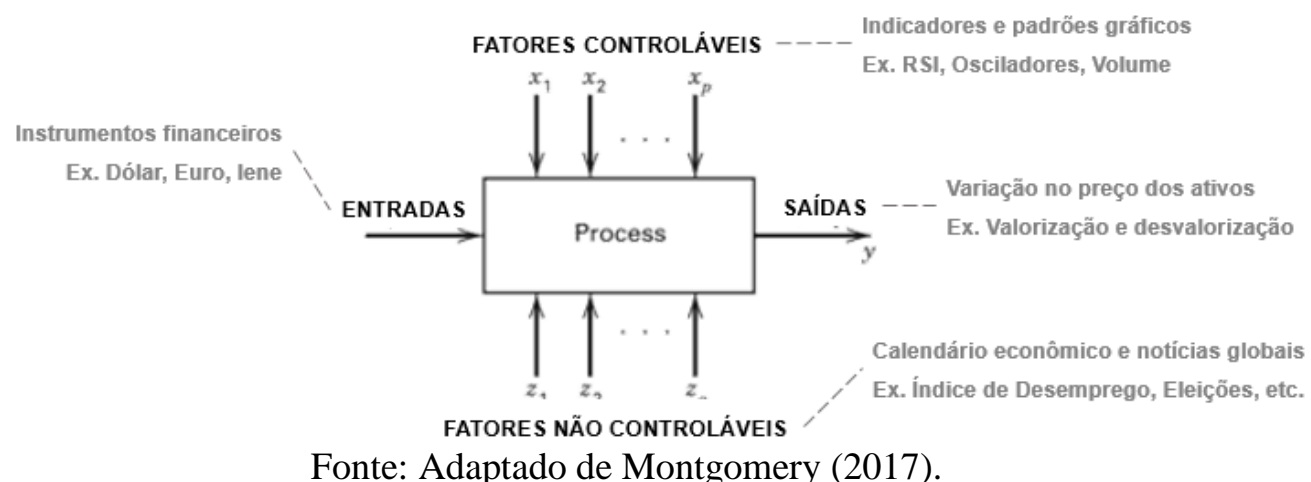

Fonte: Adaptado de Montgomery (2017). 
De acordo com Huairui Guo (2012), os principais usos do DOE são:

1. Comparação - este é um fator entre as comparações múltiplas para selecionar a melhor opção que usa o teste $\mathrm{t}-$, Teste $\mathrm{Z}$ - ou teste $\mathrm{F}$-.

2. Triagem variável - geralmente são experimentos fatoriais de dois níveis destinados a selecionar fatores importantes (variáveis) entre muitas que afetam o desempenho de um sistema, processo ou produto.

3. Identificação da função de transferência - se as variáveis de entrada importantes forem identificadas, a relação entre as variáveis de entrada e a variável de saída podem ser usadas para uma maior exploração do desempenho do sistema, processo ou produto via função de transferência.

4. Otimização do sistema - a função de transferência pode ser executada para otimização, movendo o experimento para a configuração ideal das variáveis. Desta forma, o desempenho do sistema, processo ou produto pode ser aprimorado.

5. Projeto robusto - trata da redução da variação no sistema, processo ou produto sem eliminação de suas causas. O design robusto foi iniciado pelo Dr. Genichi Taguchi, que tornou o sistema robusto contra ruído (fatores ambientais e incontroláveis são considerados ruído). Geralmente, os fatores que causam a variação do produto podem ser categorizados em três grupos principais: Externo / ambiental (como temperatura, umidade e poeira); Interno (desgaste da máquina e envelhecimento dos materiais); Variação de unidade para unidade (variações em materiais, processos e equipamentos).

\section{Mercado financeiro}

O mercado financeiro é composto por vários segmentos. Alguns exemplos são: Ações, Índices, Commodities, Fundos e Câmbio, sendo esse último o objeto de estudo desse projeto. Também conhecido como forex ou FX (do inglês Foreign eXchange), segundo Gameiro (2004), esse é o maior mercado financeiro do planeta. Enquanto o mercado de cambio opera um volume superior a \$2 trilhões de dólares por dia, no mesmo período o mercado de ações de Nova Iorque opera \$25 bilhões de dólares. No mercado de cambio, o forex e as moedas são a base de operação, ou seja, são esses instrumentos que devem ser negociados para obtenção de lucros (Calicchio, 2020).

Existem diversos fatores que indicam um possível comportamento iminente, como padrões gráficos, fatores políticos e indicadores. Um desses fatores é o indicador Relative Strength Index (RSI) ou Índice de Força Relativa IFR) que, conforme afirmado 
por Lemos (2015), é um oscilador de impulsão que mede a velocidade e a mudança dos movimentos de preços. Ele compara a variação média de preços dos períodos de avanços com a variação média dos períodos de declínios. Quando o nível desse indicador está inferior a 20, significa que há uma situação de valorização próxima, ou seja, na prática, uma compra do ativo em análise é recomendada porque o preço tende a subir. Além do RSI, outras variáveis da análise técnica podem indicar uma possível alteração no comportamento do instrumento financeiro, como indicadores de tendência, indicadores de volume, osciladores, padrões gráficos, entre outros.

Para identificar em quais situações a aplicação desses indicadores tem maiores nível de assertividade, esse trabalho propõe a aplicação do Planejamento de Experimentos, também conhecido como DoE (do inglês Design Of Experiments) (MONTGOMERY, 2009, p.01, tradução nossa).

\section{METODOLOGIA}

\section{Revisão Sistemática da Literatura (RS)}

A Revisão Sistemática (RS) é um modelo de revisão que usa métodos rigorosos e explícitos para identificar, selecionar, coletar dados, analisar e descrever as contribuições relevantes à sua pesquisa (CORDEIRO et al., 2007).

Para Donato (2019), na elaboração da RS, os seguintes passos devem estar explicitamente descritos, Quadro 1:

Quadro 1 - Etapas da Revisão Sistemática (RS).

\begin{tabular}{|l|l|}
\hline 1. Formular uma questão de investigação; & 7. Extração dos dados; \\
\hline $\begin{array}{l}\text { 2. Produzir um protocolo de investigação e } \\
\text { efetuar o seu registro (itens 1 e de } 3 \text { a 8 } \\
\text { devem constar no protocolo de elaboração da } \\
\text { revisão sistemática). }\end{array}$ & $\begin{array}{l}\text { 8. Síntese dos dados e avaliação da qualidade } \\
\text { da evidência. }\end{array}$ \\
\hline $\begin{array}{l}\text { 3. Definir os critérios de inclusão e de } \\
\text { exclusão. }\end{array}$ & $\begin{array}{l}\text { 9. Disseminação dos resultados - Publicação. } \\
\text { Todos estes passos têm de ser explicitamente }\end{array}$ \\
\hline $\begin{array}{l}\text { 4. Desenvolver uma estratégia de pesquisa e } \\
\text { pesquisar a literatura - encontrar os estudos. }\end{array}$ & 7. Extração dos dados. \\
\hline 5. Seleção dos estudos. & $\begin{array}{l}\text { 8. Síntese dos dados e avaliação da qualidade } \\
\text { da evidência. }\end{array}$ \\
\hline 6. Avaliação da qualidade dos estudos. & 9. Disseminação dos resultados - Publicação. \\
\hline
\end{tabular}

Fonte: Adaptado pelos autores, Donato (2019).

Na Figura 2, elaborou-se um fluxograma, definindo o caminho que a análise bibliométrica deste trabalho percorreu para atingir seus objetivos: 
Figura 2 - Fluxograma do estudo bibliométrico.
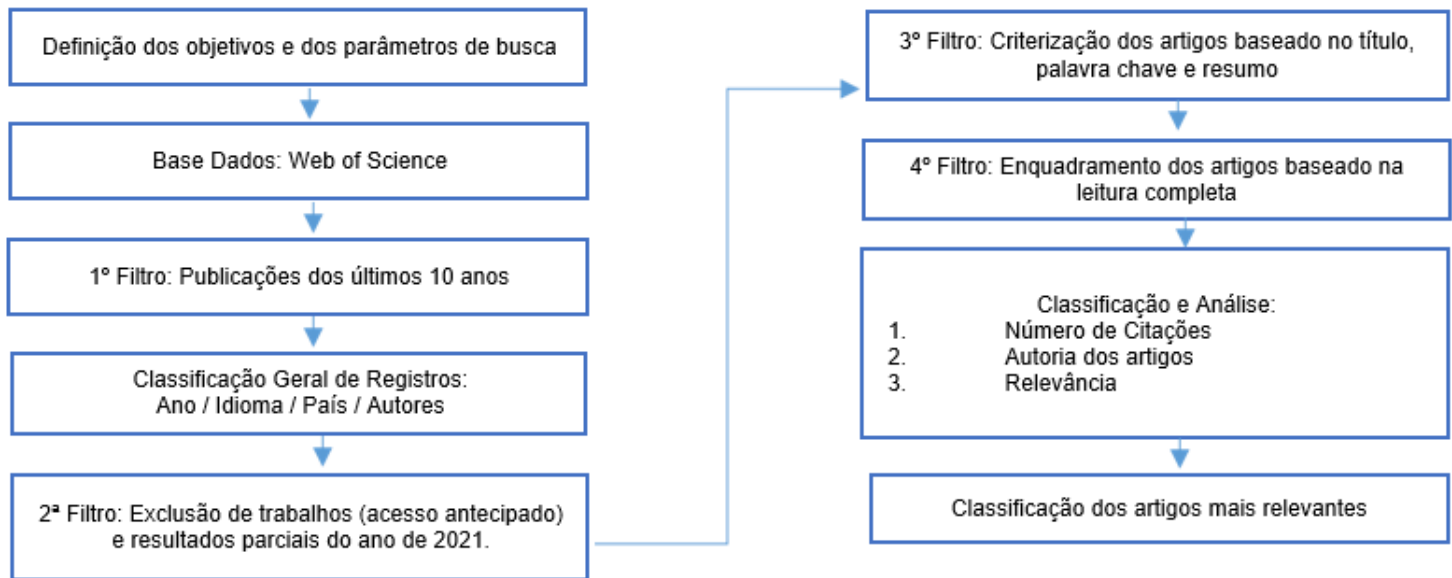

Fonte: Adaptado pelos autores de Garcia et al (2017).

Dentre as adaptações que foram feitas no quadro 1 e na figura 2, pode-se destacar como principais: a formulação da questão de pesquisa para nortear o trabalho, a definição da base de dados Web of Science e palavras chaves. O uso de critérios de inclusão e exclusão e a ênfase na avaliação e análise dos artigos identificou os trabalhos mais promissores e relevantes envolvendo DoE e Mercado Financeiro.

\section{RESULTADOS E DISCUSSÃO}

\section{Análise Bibliométrica - Base de Dados Web of Science}

Na primeira busca, aplicando o marco temporal de 10 anos foram encontrados 10.827 registros, agrupados entre Artigos Científicos, Capítulos de livros, Papel de Procedimentos; Papel de dados; Publicação Retratada; Revisões de Livros; Cartas, Resumo de reuniões, entre outros, Figura 3.

Figura 3 - Relação de publicações por idioma.

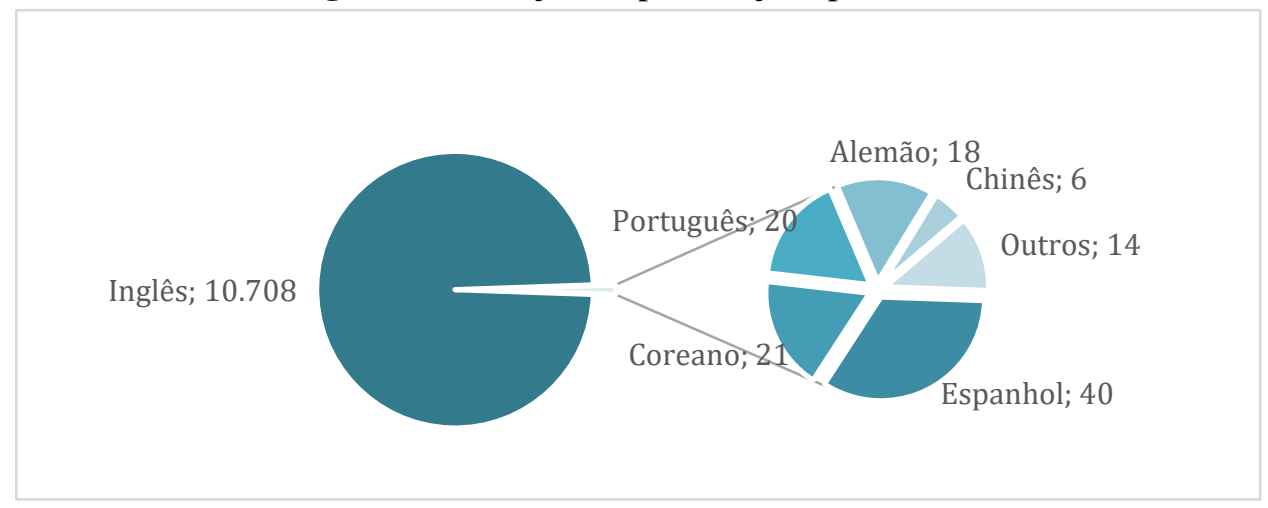

Fonte: Os autores (2021). 
Figura 4 - Relação de Publicações por ano.

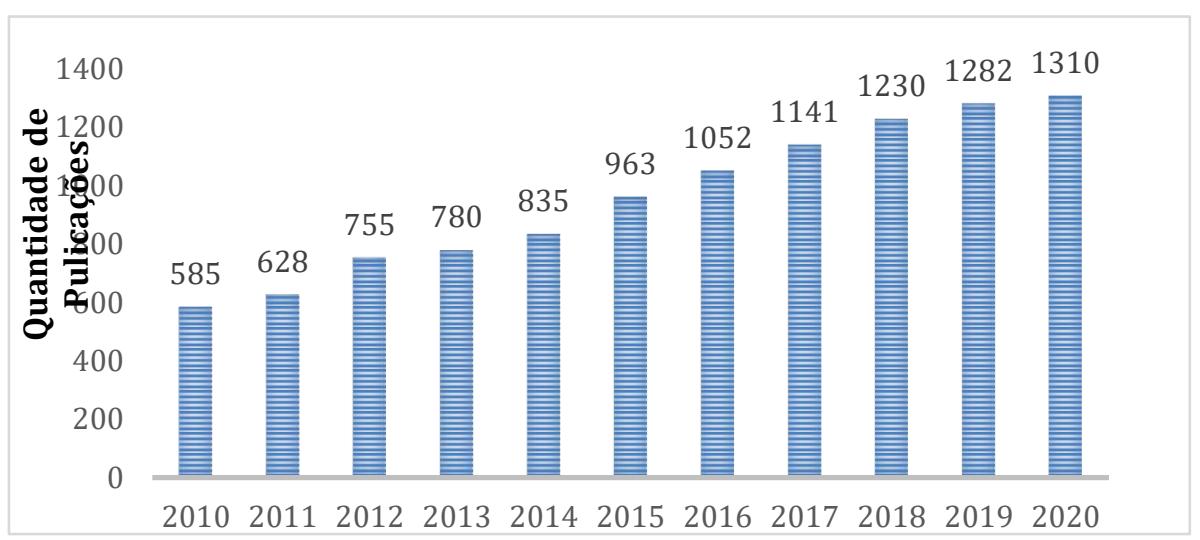

Fonte: Os autores (2021).

Ao aplicar o segundo filtro, foram excluídos trabalhos não publicados denominados (acesso antecipado) e dos resultados parciais de publicações do ano de 2021, eliminou-se 266 registros. No entanto, ainda sobraram 10.561 trabalhos para análise, Figura 4.

Observa-se um avanço das publicações, ano após ano no assunto pesquisado sobre DOE e Mercado Financeiro. No critério publicações por país, nota-se que há uma demanda significativa e de relevância nos EUA, seguido de Inglaterra, Holanda, Suíça e Alemanha. Outro fato de relevância é que se tratam de países desenvolvidos e de maior renda per capita, demonstrando que há o cuidado com a área financeira é regido por uma atenção adicional dos pesquisadores nessas nações, Figura 5.

Figura 5 - Publicações por país e suas mensurações em quantidades de publicações.

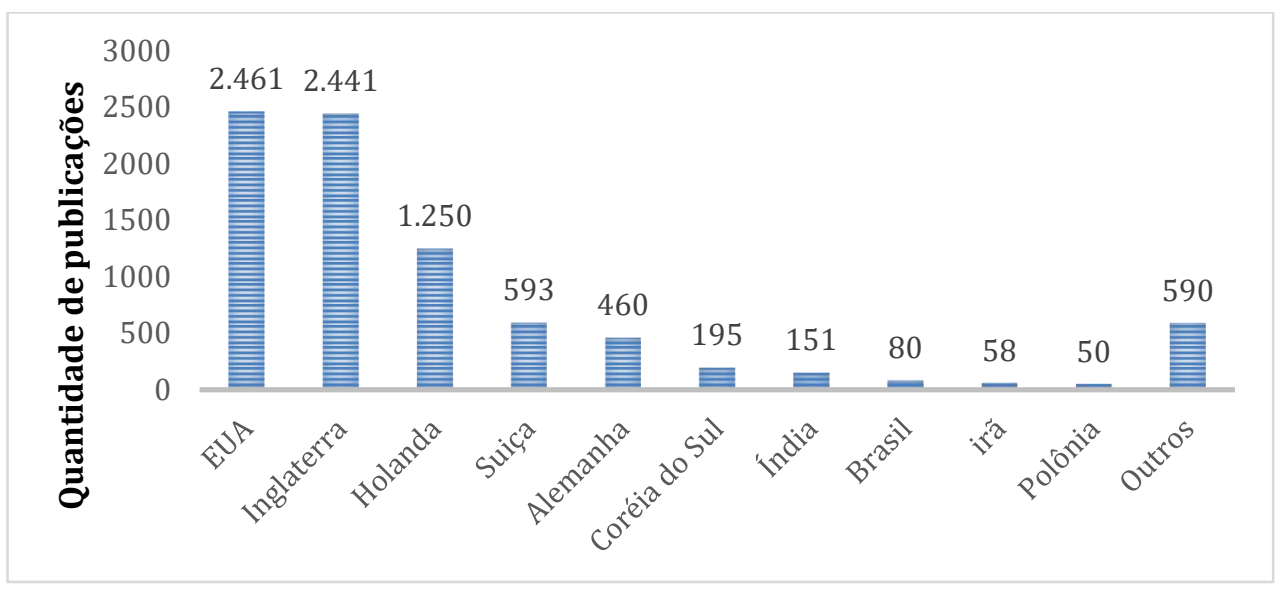

Fonte: Os autores (2021).

Quando se verifica a aplicabilidade do DoE por área, foi possível a identificação de uma quantidade razoavelmente significativa, permeando cerca de 192 duas áreas durante esse período. E das áreas que mais publicam se destacam a de Ciência de 
Materiais Multidisciplinar com 1.451 registros seguido por Engenharia Mecânica com 1.262 registros, Engenharia Química com 1.013 registros, Manufatura de Engenharia com 898 registros e Engenharia Elétrica Eletrônica com 864. Essas áreas agrupadas totalizaram 50\% de todos os registros, Quadro3:

Quadro 3 - Áreas com aplicabilidade do Design of Experiments.

\begin{tabular}{|l|c|c|}
\hline \multicolumn{1}{|c|}{ Categorias da Web of Science } & $\begin{array}{c}\text { Contagem do } \\
\text { registro }\end{array}$ & $\begin{array}{c}\mathbf{\%} \text { de } \\
\mathbf{1 0 . 8 2 7}\end{array}$ \\
\hline Ciência de Materiais Multidisciplinar & 1451 & $13 \%$ \\
\hline Engenharia Mecânica & 1262 & $12 \%$ \\
\hline Engenharia Química & 1013 & $9 \%$ \\
\hline Manufatura de Engenharia & 898 & $8 \%$ \\
\hline Engenharia Elétrica Eletrônica & 864 & $8 \%$ \\
\hline Engenharia Multidisciplinar & 662 & $6 \%$ \\
\hline Farmácia Farmacologia & 634 & $6 \%$ \\
\hline Combustíveis Energéticos & 620 & $6 \%$ \\
\hline Química Analítica & 506 & $5 \%$ \\
\hline Física Aplicada & 494 & $5 \%$ \\
\hline Sistemas de controle de automação & 463 & $4 \%$ \\
\hline Química Multidisciplinar & 460 & $4 \%$ \\
\hline Aplicações interdisciplinares da ciência da computação & 407 & $4 \%$ \\
\hline Microbiologia Aplicada à Biotecnologia & 405 & $4 \%$ \\
\hline Engenharia Industrial & 385 & $4 \%$ \\
\hline Mecânica & 373 & $3 \%$ \\
\hline Ciência de Gestão de Pesquisa Operacional & 362 & $3 \%$ \\
\hline Química Física & 357 & $3 \%$ \\
\hline Metalurgia Engenharia Metalúrgica & 333 & $3 \%$ \\
\hline Probabilidade Estatística & 328 & $3 \%$ \\
\hline Ciências ambientais & 324 & $3 \%$ \\
\hline Métodos de Pesquisa Bioquímica & 319 & $3 \%$ \\
\hline Ciência de Polímeros & 288 & $3 \%$ \\
\hline
\end{tabular}

Fonte: Os autores (2021).

Observou-se que dentre as 192 áreas, nenhuma corresponde diretamente à área financeira, demonstrando que a ferramenta de DoE é ainda subutilizada nesse setor.

\section{Análise dos Artigos - Título, Resumo e Palavras Chaves}

Ao se aplicar o terceiro filtro do processo metodológico, foi possível fazer o enquadramento dos artigos considerando como base o título, suas palavras chaves e resumo e número de citações. De um total de 10,8 mil publicações, foram identificados 22 (vinte e dois) artigos promissores, envolvendo mercado financeiro e DoE, Quadro 4. 
Quadro 4. Seleção de artigos por área, título, palavras-chave, autores e citações.

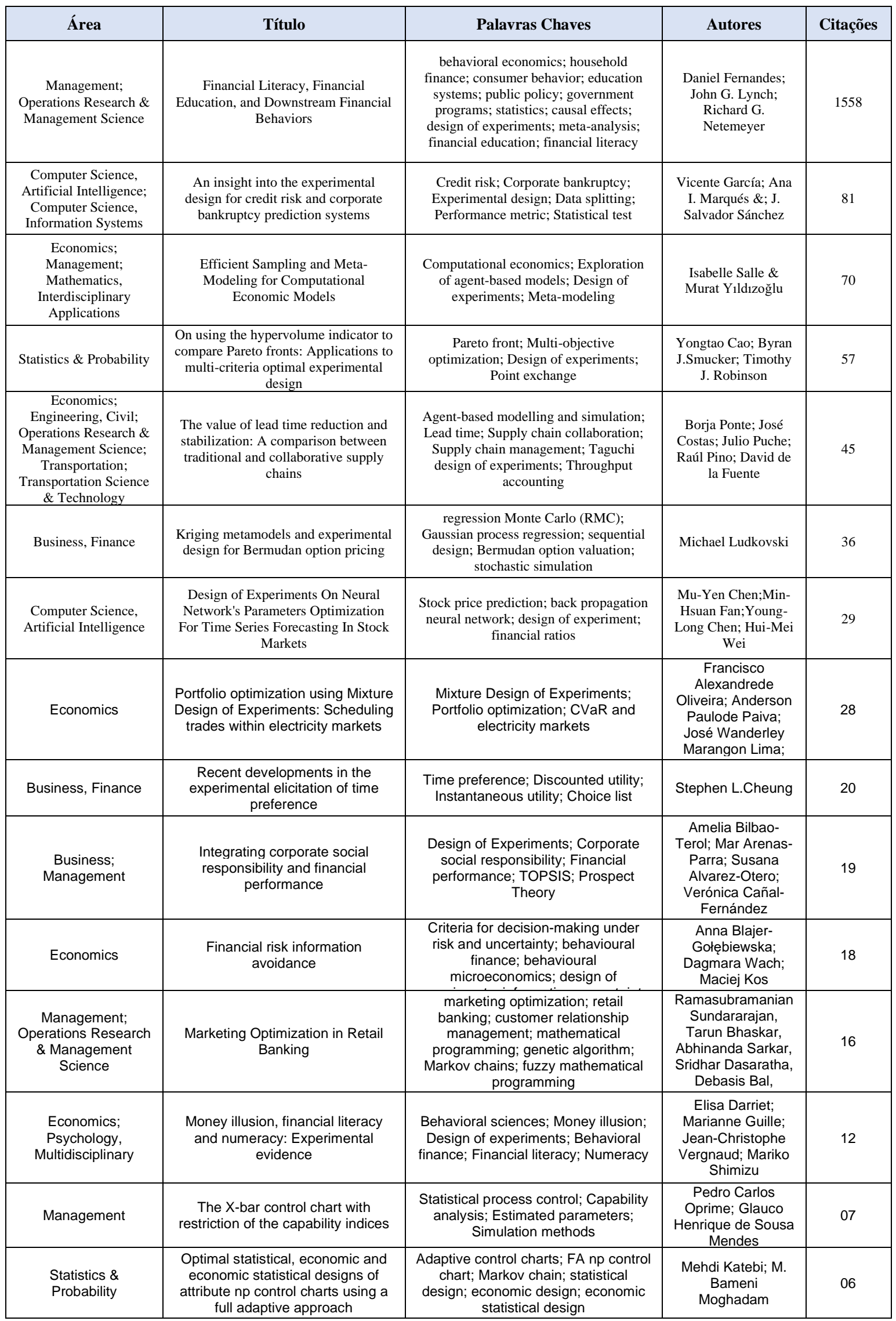




\begin{tabular}{|c|c|c|c|c|}
\hline $\begin{array}{l}\text { Statistics \& } \\
\text { Probability }\end{array}$ & $\begin{array}{l}\text { Estimating sensitivity indices } \\
\text { based on Gaussian process } \\
\text { metamodels with compactly } \\
\text { supported correlation functions }\end{array}$ & $\begin{array}{c}\text { Bayesian estimation; Computer } \\
\text { experiments; Global sensitivity } \\
\text { indices; Main-effect sensitivity } \\
\text { indices; Process-based estimator; } \\
\text { Quadrature-based estimator; Total } \\
\text { sensitivity indices }\end{array}$ & $\begin{array}{c}\text { Joshua Svenson; } \\
\text { Thomas Santner; } \\
\text { Angela Dean; } \\
\text { Hyejung Moon }\end{array}$ & 04 \\
\hline $\begin{array}{l}\text { Engineering, } \\
\text { Industrial; } \\
\text { Engineering, } \\
\text { Manufacturing }\end{array}$ & $\begin{array}{l}\text { Integrating Financial Metrics with } \\
\text { Production Simulation Models }\end{array}$ & $\begin{array}{l}\mathrm{P} \& \mathrm{Q} \text {; Discrete Event Simulation; } \\
\text { Design of Experiments }\end{array}$ & $\begin{array}{l}\text { Clive Acheson, } \\
\text { David Mackle, } \\
\text { Adrian Murphy, } \\
\text { Joseph Butterfield, } \\
\text { Peter Higgins, } \\
\text { Rory Collins, Colm }\end{array}$ & 03 \\
\hline $\begin{array}{l}\text { Mathematics; } \\
\text { Statistics \& } \\
\text { Probability }\end{array}$ & $\begin{array}{c}\text { Regular Fractions and Indicator } \\
\text { Polynomials }\end{array}$ & $\begin{array}{l}\text { Algebraic statistics; design of } \\
\text { experiments; indicator polynomial; } \\
\text { regular fractions; complex coding; } \\
\text { Galois field coding }\end{array}$ & $\begin{array}{c}\text { Giovanni Pistone } \\
\text { and Maria Piera } \\
\text { Rogantin }\end{array}$ & 03 \\
\hline $\begin{array}{l}\text { Computer Science, } \\
\text { Information Systems; } \\
\text { Computer Science, } \\
\text { Theory \& Methods }\end{array}$ & $\begin{array}{l}\text { An Exploration of the Cultivation } \\
\text { Mode of Innovation and } \\
\text { Entrepreneurship Education with } \\
\text { Modern Information Technology for } \\
\text { Statistics Students }\end{array}$ & $\begin{array}{l}\text { statistics; entrepreneurship } \\
\text { education; cultivation mode }\end{array}$ & $\begin{array}{l}\text { Yong LiHe; } \\
\text { HuangXuexin; } \\
\text { Zhou }\end{array}$ & 02 \\
\hline $\begin{array}{l}\text { Engineering, } \\
\text { Multidisciplinary }\end{array}$ & $\begin{array}{l}\text { Economic-statistical Design of NP } \\
\text { Control Chart with Variable Sample } \\
\text { Size and Sampling Interval }\end{array}$ & $\begin{array}{l}\text { The Np Control Chart; Economic- } \\
\text { statistical Design; Markov Chain; } \\
\text { Design of Experiments; Variable } \\
\text { Sampling Schemes }\end{array}$ & $\begin{array}{l}\text { M. S Fallahnezhad } \\
\text { M Shojaie-Navokh } \\
\text { Y Zare-Mehrjerdi }\end{array}$ & 02 \\
\hline Economics & $\begin{array}{l}\text { Inducing risk preferences in } \\
\text { economics experiments }\end{array}$ & experiments; risk; choice; learning & $\begin{array}{l}\text { lan M. Dobbs; } \\
\text { Anthony D. Miller }\end{array}$ & 01 \\
\hline $\begin{array}{c}\text { Engineering, } \\
\text { Industrial; Operations } \\
\text { Research \& } \\
\text { Management } \\
\end{array}$ & $\begin{array}{l}\text { The Optimal Re-sampling Strategy } \\
\text { for a Risk Assessment Model }\end{array}$ & $\begin{array}{c}\text { Risk assessment; Re-sampling } \\
\text { strategy; Imbalanced data; Design of } \\
\text { Experiments; Dual Response Surface } \\
\text { Methodology }\end{array}$ & $\begin{array}{l}\text { L. I. Tong; W. Y. } \\
\text { Wei; P. Y. Wu }\end{array}$ & 00 \\
\hline
\end{tabular}

Fonte: Os autores (2021).

Os autores que mais publicam sobre o DoE, dentre os artigos analisados são: Daniel Fernandes; John G. Lynch; Richard G. Netemeyer;Vicente García; Ana I. Marqués \&; J. Salvador Sánchez;Isabelle Salle \& Murat Yıldızoğlu;Yongtao Cao; Byran J.Smucker; Timothy J. Robinson;Borja Ponte; José Costas; Julio Puche; Raúl Pino; David de la Fuente. E, dos vinte e dois artigos elencados no quadro 4, um último filtro foi adicionado com base na leitura do resumo, identificando os artigos que efetivamente utilizaram o Design of Experiments na área do mercado financeiro, Quadro 5:

Quadro 5 - Artigos selecionados mais relevantes sobre DOE e Mercado Financeiro.

\begin{tabular}{|c|c|c|c|c|c|}
\hline $\mathbf{N}^{\mathbf{o}}$ & Título & Palavras Chaves & Autores & Citações & Disponível em \\
\hline 02 & $\begin{array}{l}\text { Uma visão sobre o desenho } \\
\text { experimental para sistemas } \\
\text { de previsão de risco de } \\
\text { crédito e falência } \\
\text { corporativa }\end{array}$ & $\begin{array}{c}\text { Risco de crédito, Falência } \\
\text { corporative, Desenho } \\
\text { experimental, Divisão de dados } \\
\text { Métrica de desempenho · Teste } \\
\text { estatístico }\end{array}$ & $\begin{array}{l}\text { Vicente García, Ana } \\
\text { I. Marques, J. } \\
\text { Salvador } \\
\text { Sanchez }\end{array}$ & 83 & $\begin{array}{l}\text { http://repositori.uji.es/ } \\
\text { xmlui/bitstream/handl } \\
\text { e/10234/161000/643 } \\
\text { 66.pdf?sequence=1\& } \\
\text { isAllowed=y }\end{array}$ \\
\hline 03 & $\begin{array}{c}\text { A estratégia de } \\
\text { reamostragem ideal para } \\
\text { um modelo de avaliação de } \\
\text { risco }\end{array}$ & $\begin{array}{c}\text { Avaliação de risco, estratégia de } \\
\text { reamostragem, dados } \\
\text { desequilibrados, design de } \\
\text { experimentos, metodologia de } \\
\text { superfície de resposta dupla }\end{array}$ & $\begin{array}{c}\text { L.I. Tong, W.Y. Wei, } \\
\text { P.Y. Wu }\end{array}$ & 35 & $\begin{array}{c}\text { https://www.research } \\
\text { gate.net/publication/2 } \\
86588710 \text { _The_opti } \\
\text { mal_re- } \\
\text { sampling_strategy_fo }\end{array}$ \\
\hline
\end{tabular}

Fonte: Os autores (2021). 
Os artigos destacados no quadro 4 registram a efetividade da aplicação do uso da ferramenta no mercado financeiro, apresentando as inúmeras possibilidades:

a) Artigo 01 (um): envolve a aplicação do DOE na previsão do mercado de ações.

b) Artigo 02 (dois) utiliza a ferramenta para construir uma base do projeto experimental para ser utilizado em aplicações de pontuação de crédito e previsão de falências no setor financeiro.

c) Artigo 03 (três) traz a preocupação dos riscos financeiros de bancos e instituições financeiras, utilizando o DOE para desenvolver um modelo de avaliação de risco.

d) Artigo 04 (quarto) usa várias técnicas, incluindo DOE, para ajudar na tomada de decisão estruturada de um banco de varejo, possibilitando a realização de análises hipotéticas e portabilidade para outros mercados e carteiras.

\section{CONSIDERAÇÕES FINAIS}

Por meio desta pesquisa pode-se ter a visualização do impacto da ferramenta de Design of Experiments no mercado financeiro que, apesar do aumento no interesse pela comunidade financeira e empresarial, infelizmente ainda é pouco utilizada.

Com a execução desta pesquisa foi possível identificar um total de 192 áreas que estão aplicando a ferramenta e que os países que mais publicam estão situados no grupo de países denominados desenvolvidos, onde a educação financeira está mais enraizada na cultura da sociedade, contando com um mercado financeiro e de capital bem desenvolvido. Identificou-se também que o idioma predominante dos artigos publicados é o inglês, seguido por espanhol.

Os autores que mais se destacaram foram Daniel Fernandes; John G. Lynch; Richard G. Netemeyer;Vicente García; Ana I. Marqués \&; J. Salvador Sánchez;Isabelle Salle \& Murat Yıldızoğlu;Yongtao Cao; Byran J.Smucker; Timothy J. Robinson;Borja Ponte; José Costas; Julio Puche; Raúl Pino; David de la Fuente.

Como trabalho futuro, fica como proposta a extensão da pesquisa, utilizando os mesmos critérios em outras bases de dados, além de uma entrevista com especialista em DOE com foco a elencar as inúmeras possibilidades que esta ferramenta pode trazer de benefícios para o mercado financeiro.

Por fim, espera-se que este trabalho possa engajar os profissionais do mercado financeiro a utilizar o DOE na identificação de probabilidades, na solução de problemas, na análise de riscos e nas tomadas de decisão. 


\section{Agradecimentos}

Os autores agradecem a empresa Social Flare.TECH (www.socialflare.tech), pelo apoio na publicação deste artigo.

\section{REFERÊNCIAS}

A. ALAFAGHANI, A. QATTAWI. Investigating the effect of fused deposition modeling processing parameters using Taguchi design of experiment method. Elsevier. Journal of Manufacturing Processes. Volume 36, December 2018, Pages 164-174. Disponível em: https://doi.org/10.1016/j.jmapro.2018.09.025. Acesso em: 20 dez. 2021.

ANTONY, JIJU. Design of Experiments for Engineers and Scientists. Elselvier Insights. 2nd Edition - February 22, 2014. Disponível em: https://books.google.com.br/books?id=p7pCAgAAQBAJ\&printsec=frontcover\&hl=pt$\mathrm{BR} \# \mathrm{v}=$ onepage \&q\&f=false. Acesso em: $10 \mathrm{dez} .2021$.

ANTONY, JIJU. A Systematic Methodology for Design of Experiments. Design of Experiments for Engineers and Scientists (Second Edition). 2014, Pages 33-50. Disponível em: https://doi.org/10.1016/B978-0-08-099417-8.00004-3. Acesso em: 10 dez. 2021.

ANTONY, JIJU. Design of Experiments and its Applications in the Service Industry. Elsevier. Design of Experiments for Engineers and Scientists (Second Edition) 2014, Pages 189-199. Disponível em: https://doi.org/10.1016/B978-0-08-0994178.00010-9. Acesso em 12 dez. 2021.

ANTONY, JIJU. 1 - Introduction to Industrial Experimentation. S. Second Edição. Oxford: Elsevier, 2014, pp. 1-6. Disponível em: https://booksite.elsevier.com/samplechapters/9780750647090/9780750647090.PDF. Acesso em: 20 dez. 2021.

ARSALAN FAROOQ, et al. An innovative approach for planning and execution of preexperimental runs for Design of Experiments. European Research on Management and Business Economics (ERMBE), Academia Europea de Dirección y Economía de la Empresa (AEDEM), vol. 22(3), pages 155-161, 2016. Disponível em:

https://econpapers.repec.org/article/idijermbe/v_3a22_3ay_3a2016_3ai_3a3_3ap_3a155 -161.htm. Acesso em: 10 dez. 2021.

B. DURAKOVIC. Design of Experiments application, concepts, examples: State of the art. Periodicals of Engineering and Natural Scince. Vol. 5 (3) (Dec. 2017) 421-439. Disponível em: http://pen.ius.edu.ba/index.php/pen/article/viewFile/145/175. Acesso em: 12 dez. 2021.

CALICCHIO, S. Forex de uma forma simples: O guia para a introdução do mercado Forex e estratégias comerciais mais eficazes no sector cambial. [s.1.]: [s.n.], 2020.

CORDEIRO, ALEXANDER MAGNO ET AL. Revisão sistemática: uma revisão narrativa. Revista do Colégio Brasileiro de Cirurgiões, v. 34, n. 6, p. 428-431, 2007. Disponível em: http://waholding.com.br/wp- 
content/uploads/revisao_sistematica_uma_revisao_narrativa.pdf. Acesso em: 05 nov. 2021.

COOK, DEBORAH J.; MULROW, CYNTHIA D.; HAYNES, R. BRIAN. Systematicreviews: synthesisofbestevidence for clinicaldecisions. Annals of internal medicine, v. 126, n. 5, p. 376-380, 1997. Disponível em:

https://www.acpjournals.org/doi/10.7326/0003-4819-126-5-199703010-00006. Acesso em: 10 nov. 2021.

C. NOVARA. Experiment design in Nonlinear Set Membership identification. American Control Conference, 2007, pp. 1566-1571. Disponívem em: https://ieeexplore.ieee.org/document/4282953. Acesso em: 15 dez. 2021.

C. SHEN, L. WANG, W. CAO, L. QIAN. Investigation of the effect of molding variables on sink marks of plastic injection molded parts using taguchi DOE Technique. Polymer-Plastics Technology and Engineering, 46 (3) (Mar. 2007) 219-225. Disponível em: https://doi.org/10.1080/03602550601152887. Acesso em: 20 jan. 2022.

DONATO, HELENA; DONATO, MARIANA. Stages for Undertaking a Systematic Review. Acta Médica Portuguesa, [S.1.], v. 32, n. 3, p. 227-235, mar. 2019. ISSN 1646-0758. Disponível em:

<https://www.actamedicaportuguesa.com/revista/index.php/amp/article/view/11923>. Acesso em: 26 set. 2021.

DURAKOVIC, BENJAMIN. Design of Experiments Application, Concepts, Examples: State of the Art. Periodicals of Engineering and Natural Scinces. International University of Sarajevo. Vol 5, n.3 pp. 421-439, 2018. ISSN 2303-4521. Disponível em: http://pen.ius.edu.ba/index.php/pen/article/viewFile/145/175. Acesso em: 27 ago. 2021.

F. KOLIVAND, R. RAHMANNEJAD. Estimation of geotechnical parameters using Taguchi's design of experiment (DOE) and back analysis methods based on field measurement data. Springer, Bulletin of Engineering Geology and the Environment, 77 (4) (2018) 1763-1779. Disponível em:

https://link.springer.com/article/10.1007\%2Fs10064-017-1042-3. Acesso em: 20 jan. 2022.

GAMEIRO, M. Forex Market: Tudo que você precisa saber começar a ganhar dinheiro. [s.l.]: [s.n.], 2004.

GARCIA, F, L., et al. Mapeamento bibliométrico da produção científica acerca da performance ambiental da manufatura aditiva. 2017. Anais do XXXVII Encontro Nacional de Engenharia de Produção - Enegep. Disponível em: 10.14488/ENEGEP2017_TN_STP_248_434_33894. Acesso em: 17 set. 2021.

HUAIRUI GUO, Ph. D. \& ADAMANTIOS METTAS. Design of Experiments and Data Analysis. Annual Reliability and Maintainability Symposium. 2012. Disponível em:

https://www.weibull.com/pubs/2012_RAMS_design_of_experiments_and_data_analysi s.pdf. Acesso em: 27 ago. 2021.

Johnson, L., Bell, G., 2009. Designed experiments in service quality applications. In: ASQ World Conference on Quality and Improvement, 18-20 May, Minneapolis, MN. 
KARIMSHOUSHTARI, MILAD; NOVARA, CARLO. Design of Experiments for nonlinear system identification: A set membership approach. Elsevier. Automatica, volume 119, September 2020, 109036. Disponível em:

https://doi.org/10.1016/j.automatica.2020.109036. Acesso em: 15 dez. 2021.

LEMOS, F. Análise Técnica dos Mercados Financeiros: Um guia completo e definitivo dos métodos de negociação de ativos. 1. Ed. São Paulo: Saraiva, 2015.

M.C. ORTIZ, M.S. SANCHEZ, L.A. SARABIA. Quality of analytical measurements: univariate regression, in: S.D. Brown, R. Tauler, B. Walczak (Eds.), Comprehensive Chemometrics: Chemical and Biochemical Data Analysis, Elsevier, Amsterdam, 2009, pp. 127e167.

MONTGOMERY, DOUGLAS C. Introdução ao controle estatístico da qualidade. 7.ed. Rio de Janeiro: LTC, 2017.

PILKINGTON, ALAN; MEREDITH, JACK. The evolution of the intellectual structure of operations management - 1980-2006: a citation/co-citation analysis. Journal of Operations Management, v. 27, p. 185-202, jun. 2009. Disponível em: https://doi.org/10.1016/j.jom.2008.08.001. Acesso em: 10 set. 2021.

SIDDAWAY AP, WOOD AM, HEDGES LV. How to do a systematic review: a best practice guide for conducting and reporting narrative reviews, meta-analyses, and metasyntheses. Annual Review of Psychology. 2019;70:747-70. Disponível em: https://www.annualreviews.org/doi/abs/10.1146/annurev-psych-010418-102803. Acesso em: 10 set. 2021.

TELFORD, JACQUELINE, K. A Brief Introduction to Design of Experiments. Johns Hopkins APL Technical Digest, Volume 27, Number 3. 2007. Disponível em: https://www.jhuapl.edu/Content/techdigest/pdf/V27-N03/27-03-Telford.pdf. Acesso em: 27 ago. 2021.

WHITFORD, WILLIAM G.; LUNDGREN, MATS; FAIRBANK, ALAIN. Cell Culture Media in Bioprocessing. Biopharmaceutical Processing, Development, Design, and Implementation of Manufacturing Processes, 2018, Pages 147-162. Disponível em: https://doi.org/10.1016/B978-0-08-100623-8.00008-6. Acesso em: 27 ago. 2021.

ZAHRAEE, S. M, ET AL. Combined Use of Design of Experiment and Computer Simulation for Resources Level Determination in Concrete Pouring Process. Jurnal Teknologi, 64(1). 2013. Disponível em: https://doi.org/10.11113/jt.v64.1315. Acesso em: 10 dez. 2021. 